\title{
Tip-Enhanced Raman Micro Spectroscopy and Imaging on Commercially Available AFM's and Raman Microscopes
}

\author{
Q. Nguyen*, J. Schreiber*, F. Adar**, A. Whitley**, R. Ossikovski*** \\ *Horiba Jobin Yvon SAS, 231, rue du Lille, 59650Villeneuve d'Ascq, France \\ **Horiba Jobin Yvon Inc., 3880 Park Ave., Edison, NJ 08820, USA \\ ***LPICM, Ecole Polytechnique, 91128 Palaiseau Cedex, France
}

Raman microscopes, as introduced in the early 1970's take full advantage of diffraction-limited optics to provide molecular and crystalline information on a size scale of the order of the laser wavelength, or smaller [1,2]. Now that the technique is well-entrenched in both the Raman and the microscopy communities, the materials characterization community is looking for higher spatial resolution. Getting beyond the diffraction limit can only be achieved by implementing some special technique like near field optics. Near field scanning microscopy has been developed by delivering and/or collecting light with a tapered optical fiber [3]. However, the optical losses in the fiber are so great, that it proved to be an impractical method for achieving near-field Raman signals. A totally different approach has been developing over the past years for collecting Raman signals from very small spots. This approach takes advantage of nanoscale manipulations of an Atomic Force Microscope (AFM) and the Surface Enhanced Raman Scattering Effect (SERS). SERS is a phenomenon discovered close to 30 years ago in which material in intimate contact with particles of noble metals (Ag, $\mathrm{Au}$ or $\mathrm{Cu}$ ) experiences an enhancement of its Raman cross-section by 6 to 14 orders of magnitude. In order to integrate AFM with SERS, the tip is coated with one of the SERSenhancing metals. Under AFM control, the tip can be moved into proximity to the sample surface and then produce a "local SERS" around the tip. This technique is called Tip Enhanced Raman Spectroscopy (TERS). The Raman signal is measured with conventional far field optics, but the difference in signal intensity with the tip close to the surface (and inside the laser's spot), and the tip withdrawn, provides a measure of the enhancement, and demonstrates sensitivity to nanoscale material. The spatial resolution, in fact, is determined by the tip-enhanced Raman signal across the sample, and is approximated by the dimension of the tip. Several academic groups have been developing this technology [5-8]. Both transmission and reflection geometries have been implemented.

In the case of crystalline silicon it is possible to design a measurement that will minimize the far field Raman signal (by controlling the orientation of the crystal's axes relative to the laser and Raman polarization) while still "allowing" the TERS signal[6]. Figure 1 illustrates a 20-fold contrast enhancement using oblique side illumination on a system composed of a LabRAM HR Raman microscope and a PSIA AFM.

The original Raman microscope was based on "global illumination" and filtered Raman images [1]. This method was found to be impractical for high-quality Raman images so state-of-the-art Raman imaging has relied on XY mapping of the sample under the microscope. This method has the important advantage of providing confocally filtered spectra. As a means of improving the performance of such systems, high quality motorized XYZ stages (with minimum movement of 0.1 micron) can now be replaced with piezo stages that provide $\mathrm{nm}$ precision and result in images with considerably better definition. But the optical images are still far field images with $\sim 0.5 \mu \mathrm{m}$ resolution. By taking advantage of the TERS systems described above, image resolution is now $100 \mathrm{~nm}$, which is determined by the tip, and is expected to improve by at least a factor of 2 to 4 . 
Figures 2 and 3 show normal Raman maps of a GaN nanowire and a structured polymer. Clearly these images recorded with far field optics and $0.1 \mathrm{~nm}$ steps will be vastly improved when recorded with TERS, using software that smoothly integrates the AFM and Raman functionalities.

\section{References}

[1] M Delhaye; P Dhamelincourt; J Raman Spectrosc. 3 (1975) 33-43

[2] Fran Adar, Andrew Whitley, Sergey Mamedov, Eunah Lee and Myriam Moreau Raman Spectroscopy and Spatial Resolution: How Small Can Raman See? Pittcon 2006

[3] E. Betzig, J.K. Trautman, T.D. Harris, J.S. Weiner, R.L. Kostelak Science 251 (1991) 14681470

[4] J. Wessel J. Opt. Society B 219851538

[5] N. Hayazawa, Y. Inouye, Z. Sekkat, and S. Kawata. Metallized tip amplification of near-field Raman scattering. Optics Comm. 183, 333-336 (2000).

[5] B. Pettinger, G. Picardi, R. Schuster, and G. Ertl. Surface enhanced Raman spectroscopy:

Towards single molecular spectroscopy. Electrochemistry (Jpn) 68(12) (2000) 942-949.

[6] D. Mehtani, N. Lee, R.D. Hartschuh, A. Kisliuk, M.D. Foster, A. P. Sokolov, and J. F. Maguire, J. Raman Spectrosc. 36 (2005) 1068-1075

[7] R. M. Stockle, Y. D. Suh, V. Deckert, and R. Zenobi. Nanoscale chemical analysis by tip-enhanced Raman spectroscopy. Chem. Phys. Lett. 318,131-136 (2000).

[8] M . S. Anderson. Locally enhanced Raman spectroscopy with an atomic force microscope. Appl. Phys. Lett. 76, 3130-3132 (20

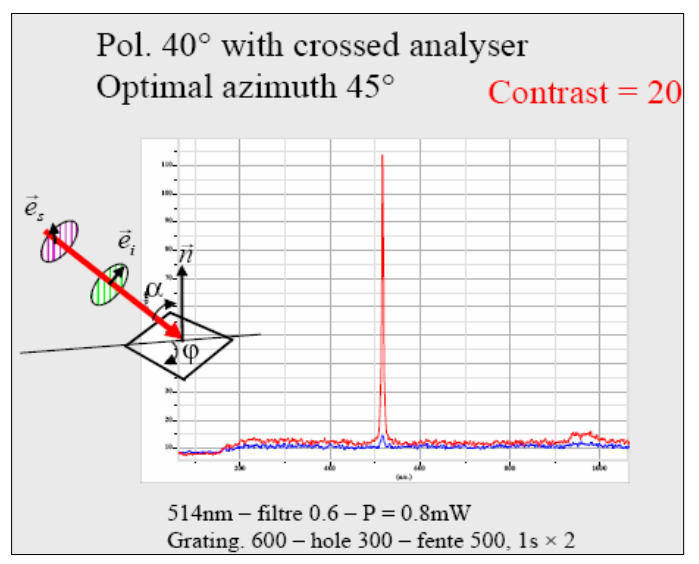

Figure 1. TERS spectrum of (001)Si recorded on a PSIA AFM and LabRAM HR with the laser at $60^{\circ}$ incident angle. Contrast was measured at 20 fold.

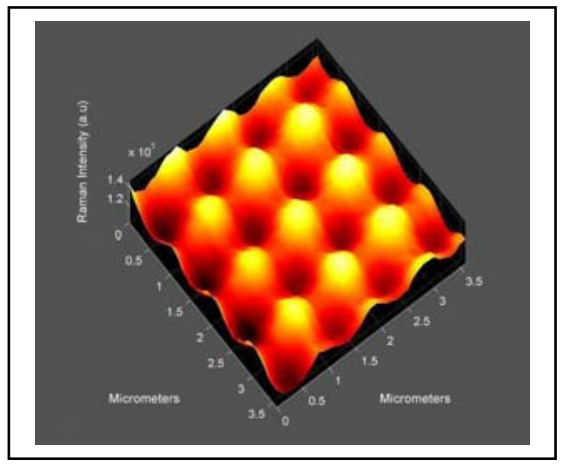

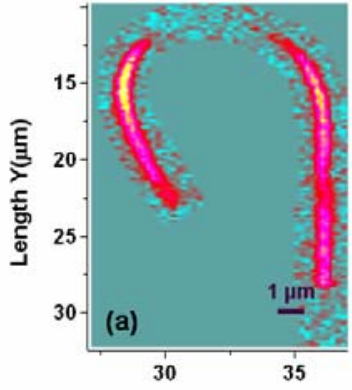
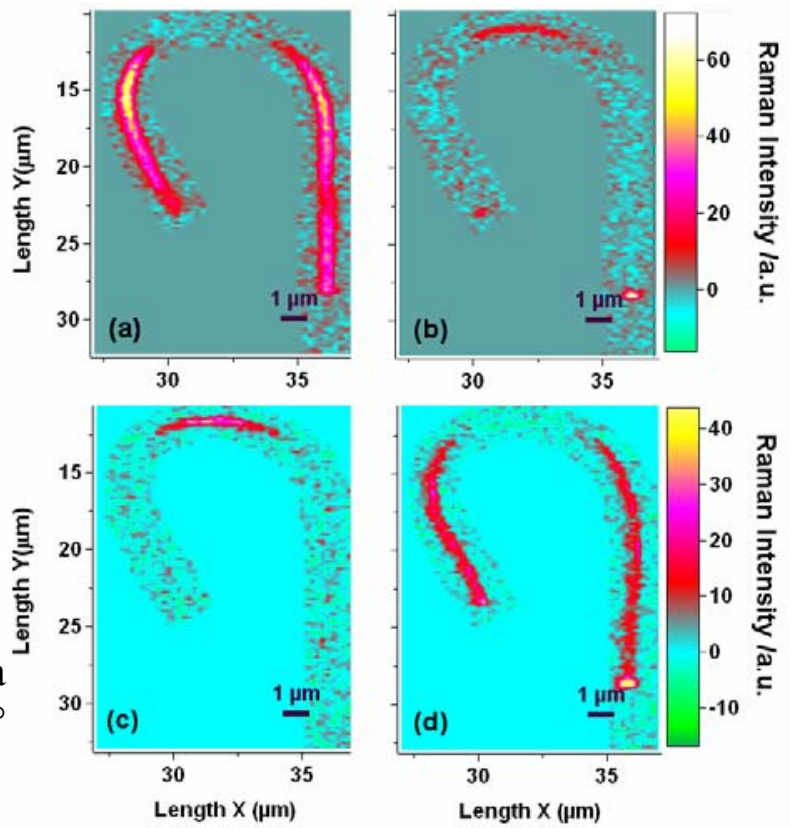

Figure 2. Far field Raman image of GaN Nanowire. Different maps were acquired under different polarization conditions.

Figure 3. Far field Raman image of structured polymer. This and the images of GaN were recorded at the University of Bordeaux by F. Lagugne and D. Talaga. 